\title{
Visual-based plant species identification from crowdsourced data
}

\author{
Hervé Goëau \\ INRIA, IMEDIA Team \\ Rocquencourt, France \\ herve.goeau@inria.fr \\ Pierre Bonnet \\ CIRAD, UMR AMAP \\ Montpellier, France \\ pierre.bonnet@cirad.fr
}

\author{
Alexis Joly \\ INRIA, IMEDIA Team \\ Rocquencourt, France \\ alexis.joly@inria.fr \\ Elise Mouysset \\ Tela Botanica Association \\ Montpellier, France \\ elise@tela-botanica.org
}

\author{
Souheil Selmi \\ INRIA, IMEDIA Team \\ Rocquencourt, France \\ souheil.selmi@inria.fr \\ Laurent Joyeux \\ INRIA, IMEDIA Team \\ Rocquencourt, France \\ laurent.joyeux@inria.fr
}

\begin{abstract}
This demo presents a crowdsourcing web application dedicated to the access of botanical knowledge through automated identification of plant species by visual content. Inspired by citizen sciences, our aim is to speed up the collection and integration of raw botanical observation data, while providing to potential users an easy and efficient access to this botanical knowledge. The result presented during the demo is an enjoying application where anyone can play to shoot fresh cut leaves and observe the relevance of species suggested in spite of various visual difficult queries.
\end{abstract}

\section{INTRODUCTION}

If agricultural development is to be successful and biodiversity is to be conserved, then accurate knowledge of the identity, geographic distribution and uses of plants is essential. Unfortunately, such basic information is often only partially available for professional stakeholders, scientists, teachers and citizens, and often incomplete for ecosystems that possess the highest plant diversity. A noticeable consequence, expressed as the taxonomic gap, is that identifying plant species is usually impossible for the general public, and often a difficult task for professionals, such as farmers or wood exploiters and even for botanists themselves. The only way to overcome this problem is to speed up the collection and integration of raw observation data, while simultaneously providing to potential users an easy and efficient access to this botanical knowledge. In this context, content-based visual identification of plant's images can be considered as one of the most promising solution to help bridging the taxonomic gap. Building effective computer vision and machine learning techniques is clearly one side of the problem. But building a real-world collaborative system allowing any user to enrich the visual botanical knowledge

Permission to make digital or hard copies of all or part of this work for personal or classroom use is granted without fee provided that copies are not made or distributed for profit or commercial advantage and that copies bear this notice and the full citation on the first page. To copy otherwise, to republish, to post on servers or to redistribute to lists, requires prior specific permission and/or a fee.

Copyright 20XX ACM X-XXXXX-XX-X/XX/XX ...\$10.00. is an even more critical one.

We developed a citizen science project around the identification of common woody species covering the Metropolitan French territory with the support of the Tela Botanica ${ }^{1}$ association, a large social botany network. Tela Botanica with both researchers and engineers involved this collaborative project, animates and promotes consistently this educational project about the French common trees identification to a larger public.

\section{MATCHING-BASED VISUAL IDENTIFI- CATION}

Technically, the first side of our application deals with content-based identification of plant leaves. State-of-theart methods addressing this objective are mostly based on leaf segmentation and boundary shape features [1, 5, 2]. Segmentation-based approaches have however several strong limitations including the presence of clutter and background information as well as other acquisition shortcomings (shadows, leaflets occlusion, holes, cropping, etc.). These issues are particularly critical in a crowdsourcing environment where we do not control accurately the acquisition protocol. Alternatively, our system is based on local features and largescale matching. Indeed, we realized that large-scale object retrieval methods [6, 4], usually aimed at retrieving rigid objects (buildings, logos, etc.), do work surprisingly well on leaves. This can be explained by the fact that even if a small fraction of the leaf remains affine invariant, this is sufficient to discriminate it from other species. Concretely, our system is based on the following steps: (i) Local features extraction (local shape \& texture features computed around Harris points) (ii) Local features nearest neighbors search with an efficient hashing-based indexing scheme (AMP-LSH [3]) (iii) Spatially consistent matches filtering with a RANSAC algorithm using an affine model (see e.g. [4]) (iv) Basic top$\mathrm{K}$ decision rule as classifier: for each species, the number of occurrences in the top- $\mathrm{K}$ images returned is used as its score.

Besides clutter robustness, the method has several advantages: it does not require any complex training phase allowing fast dynamic insertion of new crowdsourced training data, and it is weakly affected by unbalanced class distri-

\footnotetext{
$\overline{{ }^{1} \text { http: //www.tela-botanica.org/ }}$
} 


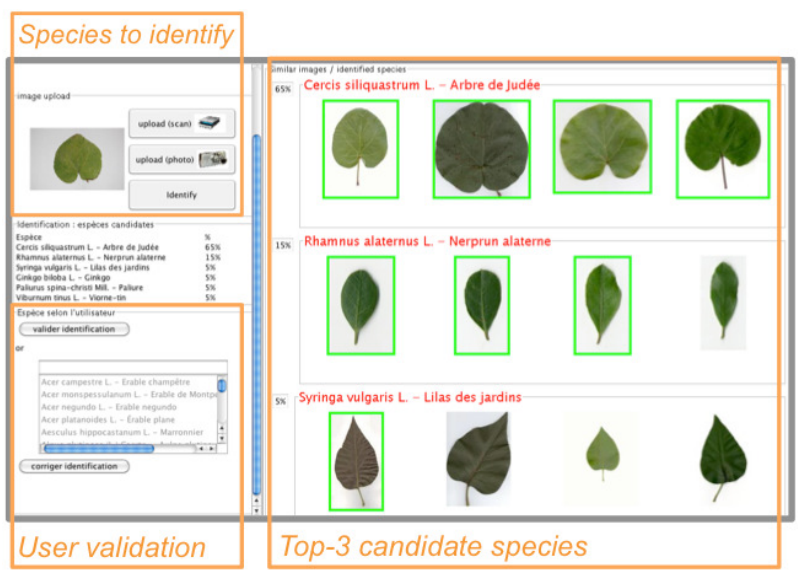

Figure 1: GUI of the web application.

bution thanks to the selectivity of the spatial consistency filtering.

\section{WEB APPLICATION AND TAG POOL- ING}

Figure 1 presents the Graphical User Interface of the web application. On the left, the user choose to load a scan or a photograph, and then, the system returns and displays on the right the top-3 species with the most similar pictures. On the bottom left part, the user can then either select and validate the top-1 suggested species, or he can choose an other species in the list, or even enter a new species name if it is not available. The uploaded image used as query is temporary stored with its associated species name. Then other users might interact with these new pictures later. So far, this last step is done offline, after that some professional botanists involved in the project validate the images and theirs species names. But, the aggregation to the visual knowledge of these uploaded images will be integrated automatically in further versions. The species names and pictures are clickable and bring the user to online taxon descriptions from the Tela Botanica web site. In this way, beyond the visual content-based recognition process, the species identification is considered as one way to access richer botanical information like species distribution, complementary pictures, textual descriptions, etc.

\section{COLLABORATIVE DATA COLLECTED}

The current data was built by several cycles of collaborative data collections and taxonomical validations. Scans of leaves were collected over two seasons, between July and September 2009 and between June and September 2010 thanks to the work of active contributors from Tela Botanica social networks. The idea of collecting only scans during this first period was to initialize the training data with limited noisy background, so that the online identification tool works sufficiently well to atract new users. Notice that this did not prevent users to submit unconstrained pictures, since our matching-based approach is relatively robust to such asymetry between training and query images. The first online application did contain 457 validated scans over 27 species and the link was mostly disseminated through Tela Botanica. It finally allowed to collect 2228 scans over 55 species. A public version of the application ${ }^{2}$ was opened in October $2010^{3}$. At the time of writing, 858 images were uploaded and tested by about 25 new users. These images are either scans or photographs with uniform background, or free photographs with natural background, and involve 15 new species from the previous set of 55 species. Note that the collected data will be used within ImageCLEF2011 plant retrieval task ${ }^{4}$.

\section{EVALUATION}

Performances, basically in terms of species identification rates, will be actually shown during the demo, with an offline version connected to a digital camera. It will consists in an enjoying demo where anyone can play to shoot fresh cut leaves. Users would notice short response times for identification (around 2 seconds), and observe relevance of species suggested in spite of the intra-species visual variability, or cases with occlusions or with non-uniform backgrounds. As a rough guide, a leave one out cross-validation (i.e. each scan used one by one as external query), gives an average precision around 0.7 over the 20 first most similar images, and gives basically the correct species as the first rank 9 times out of 10 with the knn basic rule of decision.

\section{CONCLUSIONS}

This demo represents a first step to a large scale crowdsourcing application promoting collaborative enrichment of botanical visual knowledge and its exploitation for helping users to identify biodiversity. The next version will consider a full autonomous and dynamical application integrating collaborative taxonomical validation. If the application focuses here on an educational subject, the French common trees, the performances obtained and the emulation created during this project are encouraging for addressing others floras and more narrow botanical studies.

\section{REFERENCES}

[1] P. Belhumeur, D. Chen, S. Feiner, D. Jacobs, W. Kress, H. Ling, I. Lopez, R. Ramamoorthi, S. Sheorey, S. White, and L. Zhang. Searching the worldÔs herbaria: A system for visual identification of plant species. In $E C C V$, pages 116-129. 2008.

[2] O. M. Bruno, R. de Oliveira Plotze, M. Falvo, and M. de Castro. Fractal dimension applied to plant identification. Information Sciences, 178(12):2722 - 2733, 2008.

[3] A. Joly and O. Buisson. A posteriori multi-probe locality sensitive hashing. In Proceeding of the 16th ACM international conference on Multimedia, MM '08, pages 209-218, New York, NY, USA, 2008. ACM.

[4] A. Joly and O. Buisson. Logo retrieval with a contrario visual query expansion. In Proceedings of the seventeen ACM international conference on Multimedia, pages 581-584, 2009

[5] J. C. Neto, G. E. Meyer, D. D. Jones, and A. K. Samal. Plant species identification using elliptic fourier leaf shape analysis. Computers and Electronics in Agriculture, 50(2):121 - 134, 2006.

[6] J. Philbin, O. Chum, M. Isard, J. Sivic, and A. Zisserman Object retrieval with large vocabularies and fast spatial matching. In Proceedings of the IEEE Conference on Computer Vision and Pattern Recognition, 2007.

\footnotetext{
${ }^{2}$ http://combraille.cirad.fr:8080/demo_plantscan/

${ }^{3}$ http://www.tela-botanica.org/actu/article3856.html

${ }^{4}$ http://www.imageclef .org/2011/plants
} 